\title{
Heme oxygenase and carbon monoxide protect from muscle dystrophy
}

\author{
Mun Chun Chan ${ }^{1,2}$, Olivia Ziegler ${ }^{1,2}$, Laura Liu' ${ }^{1,2}$, Glenn C. Rowe ${ }^{1,3}$, Saumya Das ${ }^{1,2}$, Leo E. Otterbein ${ }^{4}$ \\ and Zoltan Arany $^{5^{*}}$ (1)
}

\begin{abstract}
Background: Duchenne muscle dystrophy (DMD) is one of the most common lethal genetic diseases of children worldwide and is 100\% fatal. Steroids, the only therapy currently available, are marred by poor efficacy and a high side-effect profile. New therapeutic approaches are urgently needed.

Methods: Here, we leverage PGC-1a, a powerful transcriptional coactivator known to protect against dystrophy in the $m d x$ murine model of DMD, to search for novel mechanisms of protection against dystrophy.

Results: We identify heme oxygenase-1 (HO-1) as a potential novel target for the treatment of DMD. Expression of HO-1 is blunted in the muscles from the mdx murine model of DMD, and further reduction of HO- 1 by genetic haploinsufficiency worsens muscle damage in $\mathrm{mdx}$ mice. Conversely, induction of HO-1 pharmacologically protects against muscle damage. Mechanistically, $\mathrm{HO}-1$ degrades heme into biliverdin, releasing in the process ferrous iron and carbon monoxide (CO). We show that exposure to a safe low dose of $\mathrm{CO}$ protects against muscle damage in $m d x$ mice, as does pharmacological treatment with CO-releasing molecules.
\end{abstract}

Conclusions: These data identify HO-1 and $\mathrm{CO}$ as novel therapeutic agents for the treatment of DMD. Safety profiles and clinical testing of inhaled CO already exist, underscoring the translational potential of these observations.

Keywords: PGC-1a, mdx, Duchenne muscle dystrophy, Heme oxygenase, HO-1, Carbon monoxide

\section{Background}

Duchenne muscular dystrophy (DMD) is a common ( 1 in 3500 boys), X-linked recessive, inexorably lethal disease characterized by acute phases of muscular degeneration and insufficient compensatory regeneration [1]. Diagnosis occurs early in life from noticeable walking difficulty. The disease is $100 \%$ fatal. Death occurs by the early twenties from respiratory or cardiac failure caused by damage to the heart and diaphragm muscles. There is no known cure for DMD. DMD is caused by mutations in the dystrophin $(D M D)$ gene that lead to loss of functional protein. Dystrophin protein is a part of the large dystrophinglycoprotein complex that links the inner cytoskeleton of muscle cells with the extracellular basal lamina. Loss of

\footnotetext{
* Correspondence: zarany@mail.med.upenn.edu

${ }^{5}$ Cardiovascular Institute and Institute Diabetes Obesity and Metabolism,

Smilow Center for Translational Research, Perelman School of Medicine,

University of Pennsylvania, 11th floor, 3400 Civic Blvd, Philadelphia 19104, PA, USA

Full list of author information is available at the end of the article
}

dystrophin leads to dysfunction and necrosis of mature muscle cells including an increase in membrane damage from mechanical or hypo-osmotic stress and decreased function of plasma membrane proteins including neuronal nitric oxide synthase (nNOS) [1-4]. Though the genetic cause of DMD is known, how dystrophin mutations lead to muscle damage is incompletely understood, nor how to reverse the process. Significant efforts have focused recently on achieving total cure for DMD, including approaches such as exon skipping and most recently CRISPR-mediated genome editing [5-7], but significant hurdles persist. A complementary approach, which may be more imminently tractable, is the possibility of altering the natural course of the disease, via inhibiting degeneration and/or stimulating regeneration of muscle cells.

The transcriptional coactivator peroxisome proliferatoractivated receptor gamma coactivator $1-\alpha(\mathrm{PGC}-1 \alpha)$ is a powerful activator of broad metabolic programs in various tissues [8-10]. In the skeletal muscle, PGC- $1 \alpha$ strongly 
induces mitochondrial biogenesis, resulting in mice resistant to fatigue [11, 12]. A number of years ago, crossing mice that transgenically express PGC- $1 \alpha$ in the skeletal muscle into the dystrophin-deficient mice $(m d x)$ background, a well-established murine model of DMD, revealed impressive protection against dystrophy [13]. The protection has widely been presumed to result from the strong PGC-1 $\alpha$-mediated induction of neuromuscular junction genes and, in particular of utrophin, a homolog of dystrophin that is known to be protective in DMD models. However, we recently generated mice with muscle-specific expression of peroxisome proliferatoractivated receptor gamma coactivator $1-\beta$ (PGC-1 $\beta$ ), a homolog of PGC-1 $\alpha$, and found that PGC-1 $\beta$ did not induce neuromuscular junction (NMJ) genes or utrophin itself and yet was equally protective against dystrophy in the $m d x$ model [14]. This suggested that utrophin was not the key mediator of protection in the PGC- $1 \alpha$ transgenic mice. We formally tested this notion by crossing the PGC-1 $\alpha$-expressing mice into an $m d x /$ utrophin $^{-1-}$ background, which at baseline reveal much more pronounced dystrophy, including blunted growth, kyphosis, and early lethality. PGC-1 $\alpha$ significantly improved the dystrophic phenotype of these mice, including early lethality [14]. PGC- $1 \alpha$ thus potently protects against dystrophy, but not via utrophin.

How does PGC-1 $\alpha$ protect against muscle dystrophy, if not via utrophin? We investigate here two possibilities: induction of vasculature and induction of $\operatorname{Hmox} 1$, the gene encoding for heme oxygenase (HO-1).

\section{Methods}

\section{Animals}

All animal experiments were performed according to the procedures approved by the Beth Israel Deaconess Medical Center's Institutional Animal Care and Use Committee. All experimental animals used were male. C57BL/ $10 \mathrm{ScSn}-D m d^{\mathrm{mdx}} / \mathrm{J}$ mice $(m d x$ mice) were obtained from Jackson Laboratories. These mice were crossed with muscle-specific PGC- $1 \alpha$ transgenic mice (MCK-PGC- $1 \alpha$ ), described previously [11]; muscle-specific PGC-1 $\beta$ transgenic mice (MCK-PGC-1 $\beta$ ) described previously [15]; or mice with inducible PGC-1 $\alpha$ (Ind_PGC-1 $\alpha$ ) that possess the muscle-specific tetracycline-dependent activator (MCK-tTA), described previously [16], and the PGC- $1 \alpha$ coding region under control of the tet-response element promoter (TRE-PGC-1 $\alpha$ ) transgenic genes, described previously [16]. All of these mice were in C57BL/6 background; progeny were therefore in mixed C57BL/6 and C57BL/10 background. For all experiments, littermates were used as controls, and male animals were evaluated. $m d x, m d x /$ MCK-PGC- $1 \alpha, m d x /$ MCK-PGC- $1 \beta$, and $m d x /$ Ind_PGC- $1 \alpha$ were sacrificed at 9 weeks of age ( $n=5$ transgenic, 5 controls). Male $\mathrm{HMOX}^{+/-}$mice
(FVB.129S2(B6)-Hmox1 $1^{\text {tm1Poss } / J) ~ w e r e ~ o b t a i n e d ~ f r o m ~}$ Jackson Laboratories (JAX008311) and crossed with female C57BL/10ScSn-D $m d^{\mathrm{mdx} / J}$ mice. $\mathrm{HMOX}^{+/-} / m d x$ animals are therefore in mixed background, and, again, litter-mates were strictly used for control $(n=8$ experimental; 8 control). Ind_tsFLT mice, engineered to express in skeletal muscle inducible sFlt1 in a tet-OFF fashion, have been used by us previously [17] and were generated by crossing transgenic mice expressing tet-inducible sFlt1, previously described [18], with transgenic mice expressing muscle-specific expression of the tTA transactivator, as above. All doxycycline-responsive mice are in a tet-OFF system, and mice were maintained on tetracycline chow throughout pregnancy and development, and switched to regular chow at 5 weeks of age, followed by evaluation and harvesting at 9 weeks of age. Littermate controls were used for all experiments.

Protoporphyrin IX cobalt chloride (CoPP) (SigmaAldrich) was dissolved in $\mathrm{NaOH}(0.2 \mathrm{M})$ and then adjusted to $\mathrm{pH} 7.6$ with $\mathrm{HCl} .10 \mathrm{mg} / \mathrm{kg} /$ day of dissolved CoPP was injected three times a week intra-peritoneally into $\mathrm{mdx}$ male mice from 5 to 9 weeks of age $(n=10$ CoPP; 7 control). Age-matched mdx control mice were injected with $\mathrm{HCl}$-neutralized $\mathrm{NaOH}$ ( $\mathrm{pH}$ 7.6). Male $\mathrm{mdx}$ mice were exposed to carbon monoxide ( $\mathrm{CO}$ ) gas at a concentration of $250 \mathrm{ppm}$ for $1 \mathrm{~h}$ a day, 5 days a week, for 4 weeks total from 5 to 9 weeks of age. Mice were then sacrificed. Control $\mathrm{mdx}$ mice were placed in an equivalent-sized plexiglass box for $1 \mathrm{~h}$ a day ( $n=8 \mathrm{CO}, 8$ control). Male $\mathrm{mdx}$ mice were injected with $2.5 \mathrm{mg} / \mathrm{kg} /$ day of CO-releasing molecule A1 (CORM-A1) from 5 to 9 weeks of age. CORM-A1 was dissolved 5 min before injection freshly each day. Inactivated CORM-A1 was obtained by dissolving CORM-A1 and then piping nitrogen through the solution for $15 \mathrm{~min}$ to activate release of all CO. Inactivated CORM-A1 was made fresh every 2 weeks ( $n=6$ active CORM-A1; 8 inactivated CORM-A1).

\section{Cell culture}

Primary satellite cells were isolated and cultured from the entire hind limb of wild-type/control (WT) mice as previously described [19]. Cells were differentiated into myotubes using DMEM with 5\% horse serum for $72 \mathrm{~h}$. Cells were then infected with control (GFP) adenovirus (WT) or recombinant PGC- $1 \alpha$ adenovirus (Ad-PGC- $1 \alpha$ )) at multiplicity of infection of $10-30$. Ad-PGC-1 $\alpha$ contains PGC- $1 \alpha$ expressed under control of the CMV promoter and has been described previously [20]; we have used it extensively, with robust induction of PGC- $1 \alpha$ expression $[17,21,22]$. Cells were analyzed $48 \mathrm{~h}$ later.

\section{Evans blue injection and histological analysis}

For Evans blue assay, mice were injected with $1 \%$ solution i.p. at a final concentration of $1 \%$ volume to body weight 
$16 \mathrm{~h}$ priors to sacrifice. Tissues were dissected and embedded in OCT compound (VWR) and flash-frozen. Evans blue was analyzed by fluorescence microscopy. Percentage area of Evans blue extravasation in the gastrocnemius was quantified, using ImageJ $(\mathrm{NIH})$, by measuring the area of extravasation in at least ten $\times 200$ images per condition. For purposes of quantifying central nuclei and fiber sizes, sections were stained with rabbit polyclonal anti-laminin antibody (Abcam, ab11575) in order to establish fiber boundaries. We used two different staining techniques to calculate centralized nuclei: (i) frozen sections were stained with anti-laminin and counterstained with DAPI; (ii) tissue was dehydrated and embedded in paraffin and sectioned before H\&E staining. In either case, 20 images were taken at $\times 200$ and number of centralized nuclei fibers to total fibers was counted in a blinded fashion. At least 500 fibers across sections from different animals were counted for each condition. For fiber sizes, all sizes were measured using ImageJ software, and 50 bins were created spanning the smallest to largest fiber size across the two comparison groups. For each group, the \% of fibers in each bin was then calculated and plotted. Statistical comparison between groups was done using the Mann-Whitney $U$ test.

\section{Serum creatine kinase assay}

The blood was collected and serum isolated using heparincoated collection tubes, either by heart puncture or cheek bleed. Blood was collected and spun down at maximum speed in heparinized tubes, after which serum was collected, flash-frozen, and stored at -80 . Serum creatine kinase activity was then determined, simultaneously for all samples in a given experiment, with a Creatine Kinase-SL Assay Kit (Diagnostic Chemicals Limited).

\section{Real-time PCR}

Total RNA was isolated from mouse tissue using the TRIzol (Invitrogen) reagent, while RNA from cultured cells were isolated using the Turbocapture (Qiagen) method. Samples for real-time PCR analyses were reverse transcribed (Applied Biosystems), and quantitative real-time PCRs were performed on the cDNAs in the presence of fluorescent dye (SYBR green; Bio-Rad). Relative expression levels were determined using the comparative cycle threshold method. All q-RT-PCR data were normalized to the average expression of three different housekeeping genes, TATA-binding protein (TBP), hypoxanthine phosphoribosyltransferase 1 (HPRT), and $\beta$-actin, as we have described previously [23]. Sequences are provided below:

Mouse

HPRT F: GTTAAGCAGTACAGCCCCAAA

R: AGGGCATATCCAACAACAAACTT

TBP F: CCCTATCACTCCTGCCACACCAGC

R: GTGCAATGGTCTTTAGGTCAAGTTTACAGCC
B-ACTIN F: CCCTGTATGCCTCTGGTCGTACCAC

R: GCCAGCCAGGTCCAGACGCAGGATG

HMOX1 F: GGCGCACTCACCCTGAGCTGCTGG

R: CCCAGAGCTGGGCAAGGCCATGG

HMOX2 F: TCGGAGGGGGTAGATGAGTC

R: GCTTCCTTGGTCCCTTCCTT

Utrophin F: AGCCACCACATTTCGTTGGAA

R: GACTTATCGAGAGAAAGTGAGGC

VEGF-A F: GCCAGCACATAGAGAGAATGAGC

R: CGGCTTGTCACATTTTTCTGG

Cox5b F: CAGCTTGTAATGGGTTCCACAGT

R: TTTTCTCACGCGGAGCTTTC

PGC-1a F: AGCCGTGACCACTGACAACGAG

R: GCTGCATGGTTCTGAGTGCTAAG

Human

HPRT F: TGGACAGGACTGAACGTCTTG

R: CCAGCAGGTCAGCAAAGAATTTA

TBP F: GAGCCAAGAGTGAAGAACAGTC

R: GCTCCCCACCATATTCTGAATCT

B-actin F: GAGCGCGGCTACAGCTT

R: TCCTTAATGTCACGCACGATTT

HMOX1 F: GCAGAGGGTGATAGAAGAGGC

R: GATGTTGAGCAGGAACGCAGT

HMOX2 F: TCAGCGGAAGTGGAAACCTC

R: AGAAGTCCTTGACAAACTGGGT

\section{Western blotting}

Protein from gastrocnemius $(100 \mu \mathrm{g})$ was run on a $4-20 \%$ polyacrylamide gel, transferred to nitrocellulose membrane, and blotted for heme-oxygenase I using an antiHMOX1 antibody (ADI-SPA-896, Enzo Life Sciences), followed by goat anti-rabbit antibody conjugated to horseradish peroxidase (7074, Cell Signaling Technology Inc), followed by detection using ECL Reagent (Cell Signaling Technology Inc). As control, blot was stripped and probed with anti-pan-actin antibody (Cell Signaling Technology Inc). Blots were visualized and quantified with Biorad Image-Lab Software.

\section{Patient biopsies}

Biopsies from patients were kindly obtained from the lab of Dr. Louis Kunkel. Biopsies were taken of the vastus lateralis of normal controls and latissimus dorsi of DMD muscle. The WT biopsies were from healthy male subjects ranging from 13 to 32 years of age. Biopsies from DMD patients were from 12 to 20 years of age with different genetic defects in the dystrophin gene.

\section{Statistical analysis}

The data are presented as means \pm SEM. Statistical analysis was performed with Student's $t$ test for all in vitro experiments and ANOVAs for all in vivo experiments. 
Fiber size comparisons were done using the MannWhitney $U$ test. $P$ values of $<0.05$ were considered statistically significant.

\section{Results}

Inhibition of VEGF signaling worsens dystrophy

We showed a number of years ago that PGC- $1 \alpha$ powerfully induces angiogenesis in the skeletal muscle [21, 24]. The new vessels are functional, patent, non-leaky, and carry blood [25]. PGC-1 $\alpha$ thus coordinately induces the consumption of fuel and oxygen (in mitochondria) with the delivery of fuel and oxygen (via blood vessels). Reduced blood flow and ischemia have been proposed to play an important role in DMD models, in part leading to the rationale for the recent use of the vasodilators sildenafil and tadalafil in clinical trials (with, thus far, mixed results). We therefore reasoned that perhaps, PGC- $1 \alpha$ exerts its potent anti-dystrophic effects via dramatic increase in vascular density and blood flow. To test this notion, we first generated, in the $m d x$ background, mice that express, in the muscle only and in a tetracycline-inducible manner, a locally secreted soluble VEGF receptor 1 (also known as soluble Fms-like tyrosine kinase 1, sFlt1). This VEGF "trap" neutralizes extracellular VEGF signaling and blocks angiogenesis. These mice, which we name Ind_tsFLT, were generated by breeding transgenic mice expressing tetracyclineinducible sFlt1, a kind gift of Eli Keshet [18]), with transgenic mice expressing muscle-specific expression of the tTA transactivator, also previously described [16], all in the mdx background (Fig. 1a). Thus, the mice express the VEGF trap only in the skeletal muscle and only in the absence of doxycycline. Mice were fed doxyclycinecontaining chow throughout pregnancy, development, and weaning, to maintain the VEGF trap suppressed. At 5 weeks of age, mice were changed to normal chow, leading to robust expression of the VEGF trap in the skeletal muscle (Fig. 1b). Importantly, by virtue of a heparin-binding domain, the VEGF trap does not leak into the circulation or to other tissues (Fig. 1b), as originally reported [18]. Littermate mice that did not contain the sFLT transgene were used as controls and were similarly maintained on doxy-chow and released to normal chow at 5 weeks, thus controlling for any effects of doxycycline chow.

$M d x$ mice undergo cycles of muscle degeneration and regeneration, most marked during the first 3-10 weeks of life. Muscle degeneration can be detected during this period in a number of fashions, including accumulation of muscle creatine kinase (CK) in serum, and extravasation of Evans blue dye, which normally remains intravascular in the absence of tissue damage. Strikingly,
A

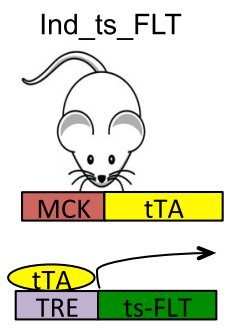

D

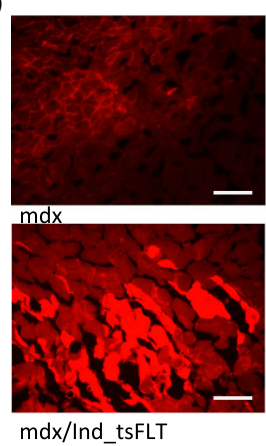

B

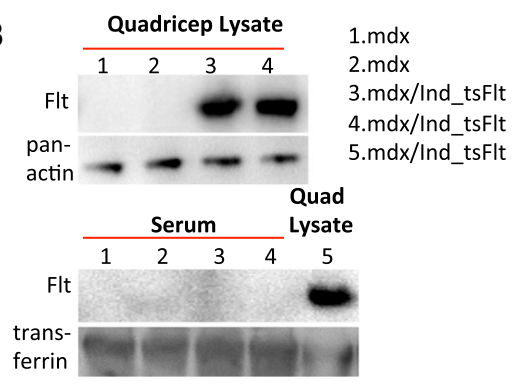

E
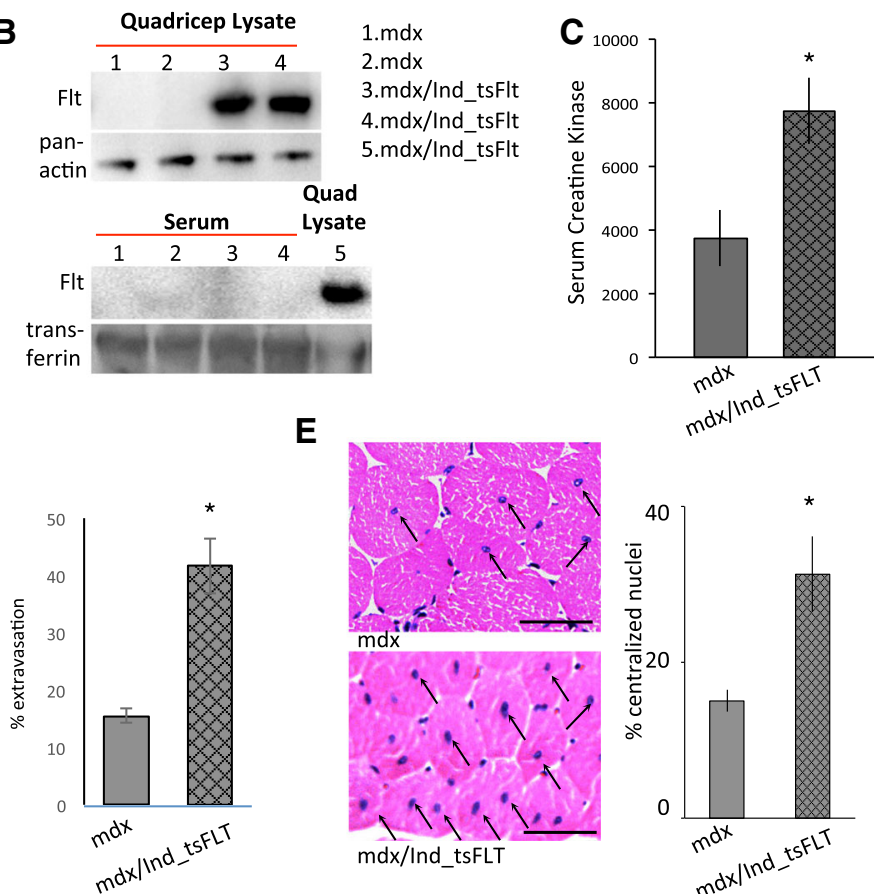

Fig. 1 Inhibition of VEGF signaling worsens dystrophy. a Dual transgenic system to express both sFlt 1 in the muscle only and only in absence of doxycycline. MCK muscle creatine kinase promoter, tTA tetracycline-suppressed transactivator, TRE tTA-responsive promoter. b Western blotting of sFlt1 demonstrating induction of sFlt1 in the muscle (top) and absence of spilling of sFlt1 into the circulation (bottom). c-e Serum creatine kinase levels (c), \% Evans blue dye extravasation (d), and \% central nuclei in gastrocnemius muscle (e) from mdx mice bearing the indicated transgenes. Scale bar $=50 \mu \mathrm{m} . n=8 .{ }^{*} P<0.05$, Student's $t$ test. Error bars indicate \pm SEM 
induction of the VEGF trap in the skeletal muscle of $m d x$ mice led to a near doubling of serum CK levels (Fig. 1c). This was accompanied by marked increase in EBD extravasation (Fig. 1d). Following muscle degeneration, cycles of regeneration are driven by the activation of resident progenitor cells and their fusion with degenerating myofibers. This process can be detected by the appearance and accumulation of central nuclei, which otherwise normally reside in the periphery of fibers, as well as the accumulation of small regenerated myofibers. Consistent with the increase in muscle damage, induction of the VEGF trap in the skeletal muscle of $m d x$ mice markedly increased the accumulation of central nuclei (Fig. 1e). These data demonstrate that suppression of VEGF signaling in $m d x$ mice markedly worsens muscle dystrophy in this model, consistent with the notion that impaired vascular homeostasis worsens dystrophy.

\section{PGC-1a protects against dystrophy independently of angiogenesis}

Can suppression of VEGF signaling abrogate the ability of PGC- $1 \alpha$ to improve muscle dystrophy? To test this, we used a triple transgenic model, in the background of the $m d x$ model, whereby both PGC- $1 \alpha$ and the VEGF trap are simultaneously induced in the skeletal muscle upon removal of doxycycline (Fig. 2a). This was achieved by crossing Ind_tsFlt mice with mice containing a third transgene encoding for tetracycline-inducible PGC-1 $\alpha$, a kind gift from Dr. Dan Kelly [16]. Therefore, in these triple transgenic mice, the VEGF trap and PGC- $1 \alpha$ are simultaneously induced, only in the skeletal muscle and only in the absence of doxycycline. Induction of PGC- $1 \alpha$ alone (without the VEGF trap) led to a robust induction of vascular density, which was completely abrogated with coinduction of the VEGF trap (Fig. 2b), as we have shown before in a non-dystrophic context [17], thus demonstrating the effectiveness of suppression of angiogenesis in this model. We therefore used this system to test the hypothesis that PGC-1 $\alpha$ improves muscle dystrophy by virtue of stimulating vascular density. If true, then induction of PGC- $1 \alpha$ in the context of simultaneously induced VEGF trap should show no improvement compared to the VEGF trap alone. Strikingly, however, despite the complete block in angiogenesis, the induction of PGC- $1 \alpha$ was still able to protect against dystrophy in the presence of the VEGF trap, as shown by reduction of circulating CK (Fig. 2c), reduced extravasation of EB dye (Fig. 2d), and reduced accumulation of central nuclei (Fig. 2e). Thus, these data show
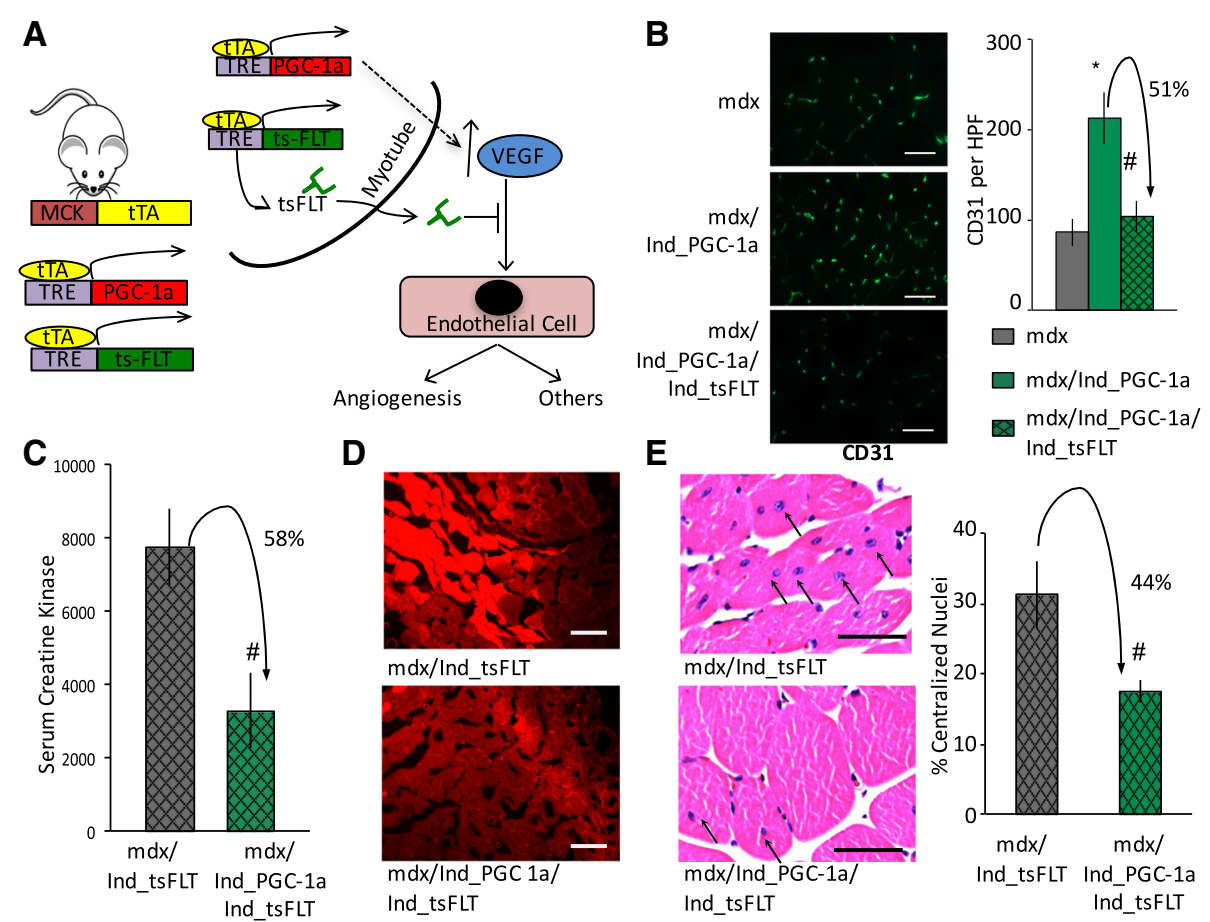

Fig. 2 PGC-1a protects against dystrophy independently of angiogenesis. a Tri-transgenic system to express both PGC-1a and sFlt1 in the muscle only and only in absence of doxycycline. MCK muscle creatine kinase promoter, tTA tetracycline-suppressed transactivator, TRE tTA-responsive promoter. b PGC-1a-induced angiogenesis is blocked in the presence of sFlt1: capillary density, identified by CD31 staining, in muscle cross sections from $\mathrm{mdx}$ mice bearing the indicated transgenes; quantification is on the right. c-e Despite absence of angiogenesis, PGC-1 a protects against dystrophy: serum creatine kinase levels (c), sample Evans blue staining in diaphragm (d), and \% central nuclei in gastrocnemius muscle (e) from $m d x$ mice bearing the indicated transgenes. $n=8$. ${ }^{*} P<0.05$ versus $m d x$ alone, ${ }^{\#} P<0.05$ versus mdx/Ind_PGC-1a, Student's $t$ test. Scale bar $=50 \mu \mathrm{m}$. Error bars indicate \pm SEM 
that suppression of VEGF signaling in $m d x$ mice does not prevent PGC-1 $\alpha$ from protecting against muscle dystrophy in this model.

\section{HO-1 expression is reduced in dystrophic muscle and is rescued by PGC-1a}

How does PGC-1 $\alpha$ protect against muscle dystrophy, if not via utrophin, via NMJ induction, or via increases in vascular density? We reasoned that identifying other pathways induced by PGC- $1 \alpha$ may point to novel pathways involved in protection against dystrophy. In search of genes induced by PGC- $1 \alpha$ that may have protective roles in the skeletal muscle, our attention was caught by Hmox 1 , the gene encoding for heme oxygenase (HO- 1 ), as a gene of interest. HO-1 degrades the cyclic prosthetic group heme, liberating ferrous iron and yielding biliverdin and gaseous carbon monoxide $(\mathrm{CO})$ in the process. Heme is abundant in the skeletal muscle, present for example in myoglobin and mitochondrial cytochromes. A growing literature supports a protective role for $\mathrm{HO}-1$ in various contexts, though it remains unclear to what extent these benefits accrue from the degradation of toxic heme, the generation of anti-oxidant biliverdin, and/or protective effects of CO [26-28]. We therefore decided to focus attention on $\mathrm{HO}-1$. Interestingly, the Kunkel lab recently identified small molecule inducers of $\mathrm{HO}-1$ as protective in a zebrafish model of DMD, although subsequent experiments raised into question if protection involved increasing $\mathrm{HO}-1$, as originally proposed $[29,30]$.

Consistent with previous reports, we find induction of mitochondrial (cox5b) and angiogenic (VEGFA) genes,

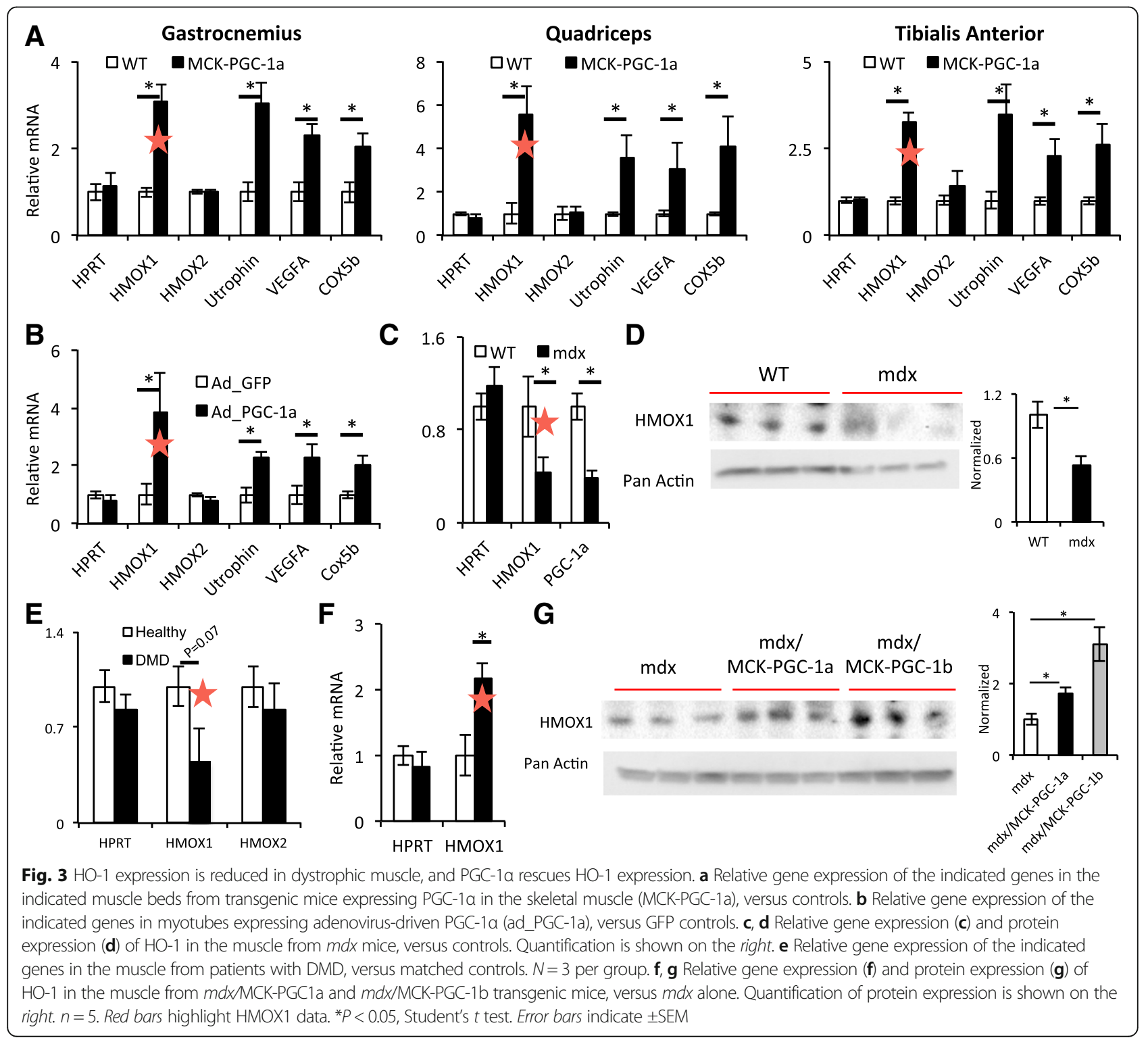


and utrophin, in quadriceps, gastrocnemius, and tibialis anterior, of mice that transgenically express PGC- $1 \alpha$ in the skeletal muscle under control of the muscle creatine kinase promoter (MCK-PGC-1 $\alpha$ mice) (Fig. 3a). In addition, we noted a strong threefold induction of Hmox1. Adenoviral delivery of PGC-1 $\alpha$ to differentiated primary myotubes induced Hmox 1 fourfold (Fig. 3b), indicating that the induction of Hmox 1 by PGC- $1 \alpha$ is cell autonomous. To assess the role for $\mathrm{HO}-1$ in muscle dystrophy, we first tested its expression in the muscle from $m d x$ mice. As shown in Fig. 3c, d, both transcript and protein expression of $\mathrm{HO}-1$ were depressed more than $50 \%$ in the muscle from these mice, compared to agematched, sex-matched, C57/BL10 mice, purchased from the same vendor and bred and maintained in the same mouse facility and suite as the mdx mice. Evaluation of human muscle biopsies revealed a strong trend for depressed expression of $\mathrm{HO}-1$ (Fig. 3e, $P<0.07$ ) (although these data must be interpreted with caution, in light of small number of samples and variability of biopsy site).
Transgenic expression of PGC- $1 \alpha$ in the $m d x$ background reversed this inhibition, inducing Hmox 1 transcript and HO-1 protein more than twofold compared to littermate controls not expressing the transgene (Fig. 3f, g). Transgenic expression of PGC-1 $\beta$ in the skeletal muscle also induced HO-1 more than twofold in $m d x$ mice compared to littermate controls not expressing the transgene (Fig. 3g). These data indicate that HO-1 expression is depressed in muscle dystrophy and that induction of PGC-1 $\alpha$ can reverse the repression of $\mathrm{HO}-1$.

Increasing HO-1 expression ameliorates muscle damage, while decreased HO-1 expression exacerbates muscle damage in $\mathrm{mdx}$ mice

In light of the known beneficial effects of PGC- $1 \alpha$ in $m d x$ mice, the data above suggested that induction of HO-1 may improve dystrophy in this model. To test this notion, we treated $m d x$ mice with cobalt protoporphyrin (Co-PP), a well-established and powerful inducer of HO-1 expression [31]. Treatment of $m d x$ mice with

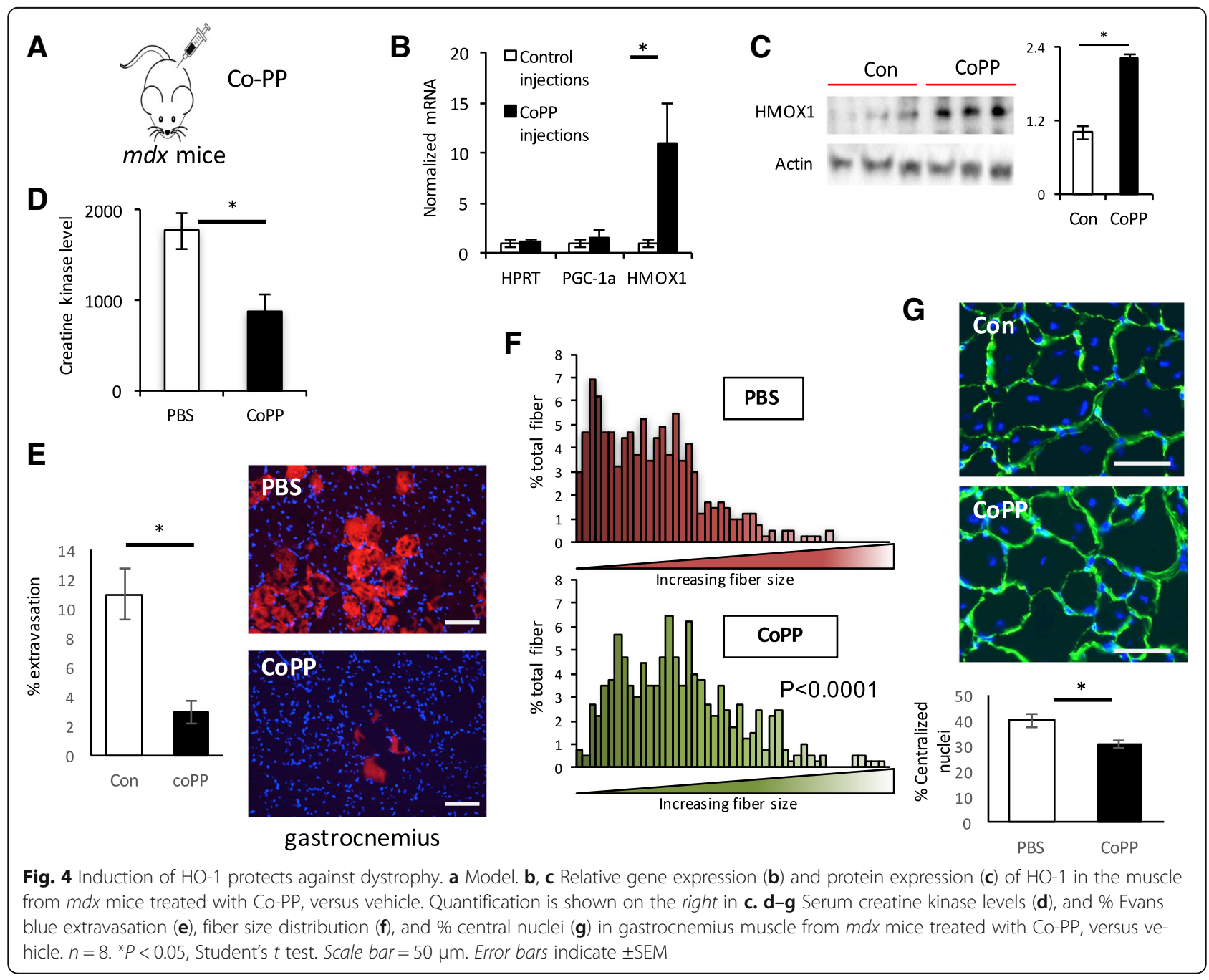


injections of Co-PP three times a week for 4 weeks led to robust induction of HO-1 mRNA and protein expression measured in the gastrocnemius muscle (Fig. $4 \mathrm{a}-\mathrm{C}$ ). Strikingly, accumulation of $\mathrm{CK}$ in the serum of $m d x$ mice was reduced by $50 \%$ (Fig. $4 \mathrm{~d}$ ) by treatment with Co-PP. This was accompanied by visible inhibition of Evans blue dye extravasation in the diaphragm and gastrocnemius (Fig. 4e). Consistent with the inhibition of muscle damage, treatment of $m d x$ mice with Co-PP preserved larger myofiber size (Fig. 4f) and markedly reduced the accumulation of central nuclei (Fig. 4g). These data demonstrate that treatment with $\mathrm{Co}-\mathrm{PP}$ and induction of $\mathrm{HO}-1$ in $m d x$ mice markedly improves muscle dystrophy in this model.

The data above indicate that induction of HO-1 is sufficient to protect against muscle dystrophy. To test if HO-1 is also necessary for endogenous adaptation to dystrophy, we crossed mice heterozygous null at the Hmox 1 locus into the $m d x$ background (Fig. 5a). Mice lacking HO-1 have been described [32]. Homozygous mice are sometimes viable but display numerous phenotypes, including anemia, abnormal iron storage and metabolism, and chronic inflammation. On the other hand, heterozygous mice are largely normal at baseline. As shown in Fig. 5b, c, the expression of HO-1 mRNA and protein is decreased by $50 \%$ in heterozygous mice, indicating haploinsufficiency at this locus. The mice are thus useful to test the role of HO- 1 in adaptation to the $m d x$ background. No abnormalities were seen in the muscle from $H_{m o x} 1^{+/-}$mice at baseline. On the other hand, as shown in Fig. 5d, accumulation of $\mathrm{CK}$ in the serum was significantly worsened in $H m o x 1^{+/-} / m d x$ mice, compared to $m d x$ alone. This was accompanied by visibly more Evans blue dye extravasation in the diaphragm and gastrocnemius (Fig. 5e). Evidence of compensatory increases in regeneration was seen with significantly smaller fiber sizes (Fig. 5f) and significantly more central nuclei (Fig. 5g). Haploinsufficiency at the Hmoxl locus thus worsens dystrophy in $m d x$ mice, indicating that $\mathrm{HO}-1$ is required for adaptations to the $m d x$ background.

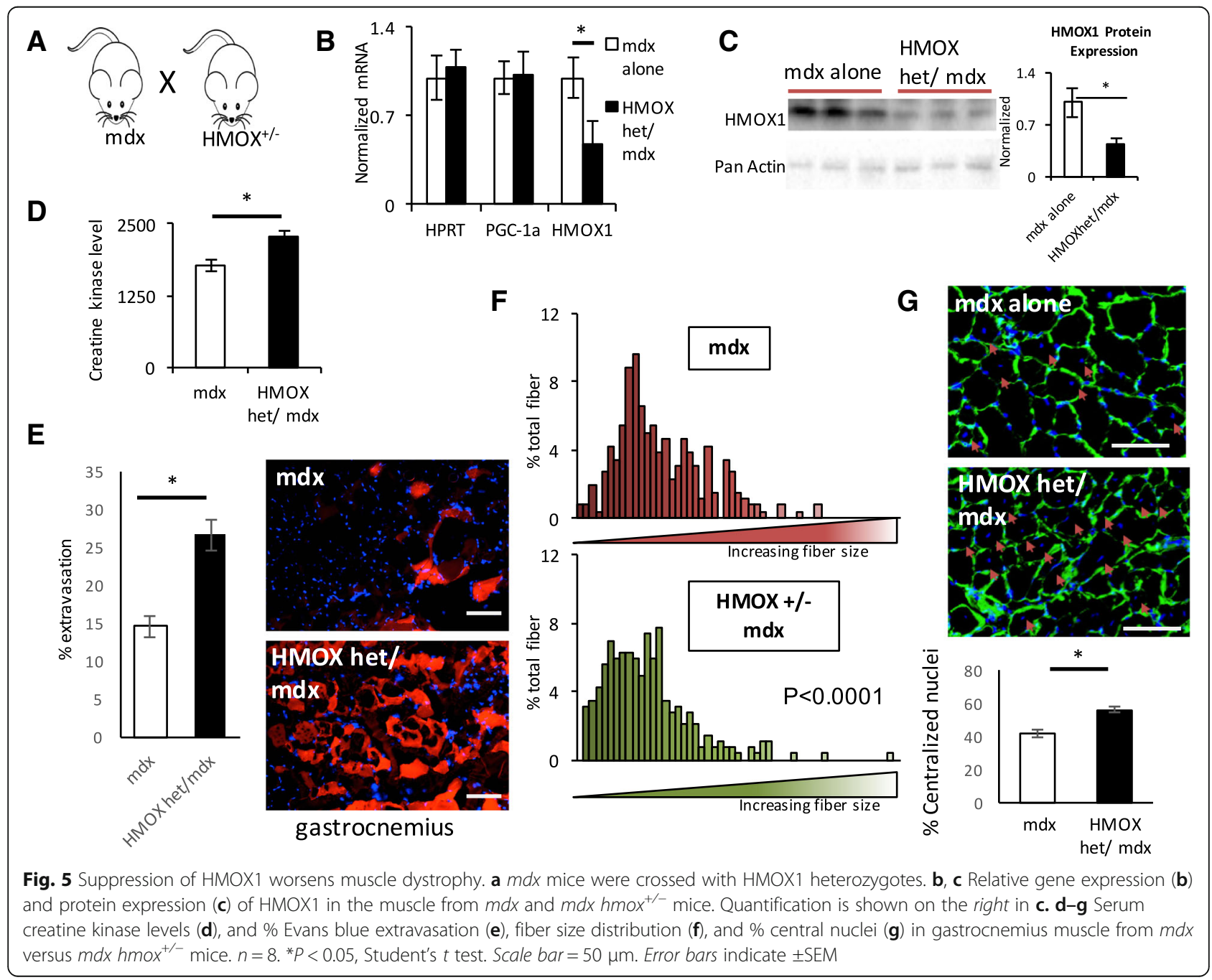


Carbon monoxide treatment ameliorates muscle damage in $\mathrm{mdx}$ mice

By what mechanism does $\mathrm{HO}-1$ improve dystrophy? HO-1 degrades the cyclic prosthetic group heme, liberating ferrous iron and yielding biliverdin and $\mathrm{CO}$ gas in the process (Fig. 6a). The benefits of HO-1 could in theory accrue from the degradation of toxic heme, the generation of anti-oxidant biliverdin, and/or protective effects of $\mathrm{CO}$ itself. We therefore tested if delivery of $\mathrm{CO}$ alone to $m d x$ mice could confer protection against dystrophy. We achieved delivery of $\mathrm{CO}$ by two methods: first, inhalation of low-dose $\mathrm{CO}$ (250 ppm, 0.025\%) and, second, by using CO-releasing molecules (CO-RMs). CORM-A1 is a water soluble $\mathrm{CO}$-releasing molecule with a relatively long half-life (21 $\mathrm{min}$ at $37{ }^{\circ} \mathrm{C}, \mathrm{pH} 7.4$ [33]). Exposure of $m d x$ mice to ambient low-dose $\mathrm{CO}$ for $1 \mathrm{~h} /$ day for 4 weeks led to a dramatic improvement in dystrophy, evidenced by marked improvement of Evans blue extravasation in diaphragm, and a $40 \%$ reduction in circulating creatine kinase levels (Fig. 6b-d). Hmox1 expression was not induced by $\mathrm{CO}$ (Fig. 6e), consistent with mechanistic activity downstream of $\mathrm{HO}-1$. Interestingly, myofiber sizes were not altered with $\mathrm{CO}$ exposure (Fig. 6f). In addition, central nuclei were slightly increased (Fig. 6g), suggesting that CO may also affect myoprogenitor proliferation or differentiation.

Treatment of $m d x$ mice with daily injections of CORM-A1 for 4 weeks recapitulated all of the findings seen with inhaled $\mathrm{CO}$, including marked improvements in dystrophy, with a dramatic reduction of Evans blue extravasation, and reduced circulating CK levels (Fig. 7a-c), unaltered expression of Hmoxl (Fig. 7d), unaltered myofiber sizes (Fig. 7e), and slight increase in central myonuclei (Fig. 7f).

Together, these data indicate that delivery of $\mathrm{CO}$, either via inhalation or via pharmaceutical delivery, strongly improves indices of muscle dystrophy.

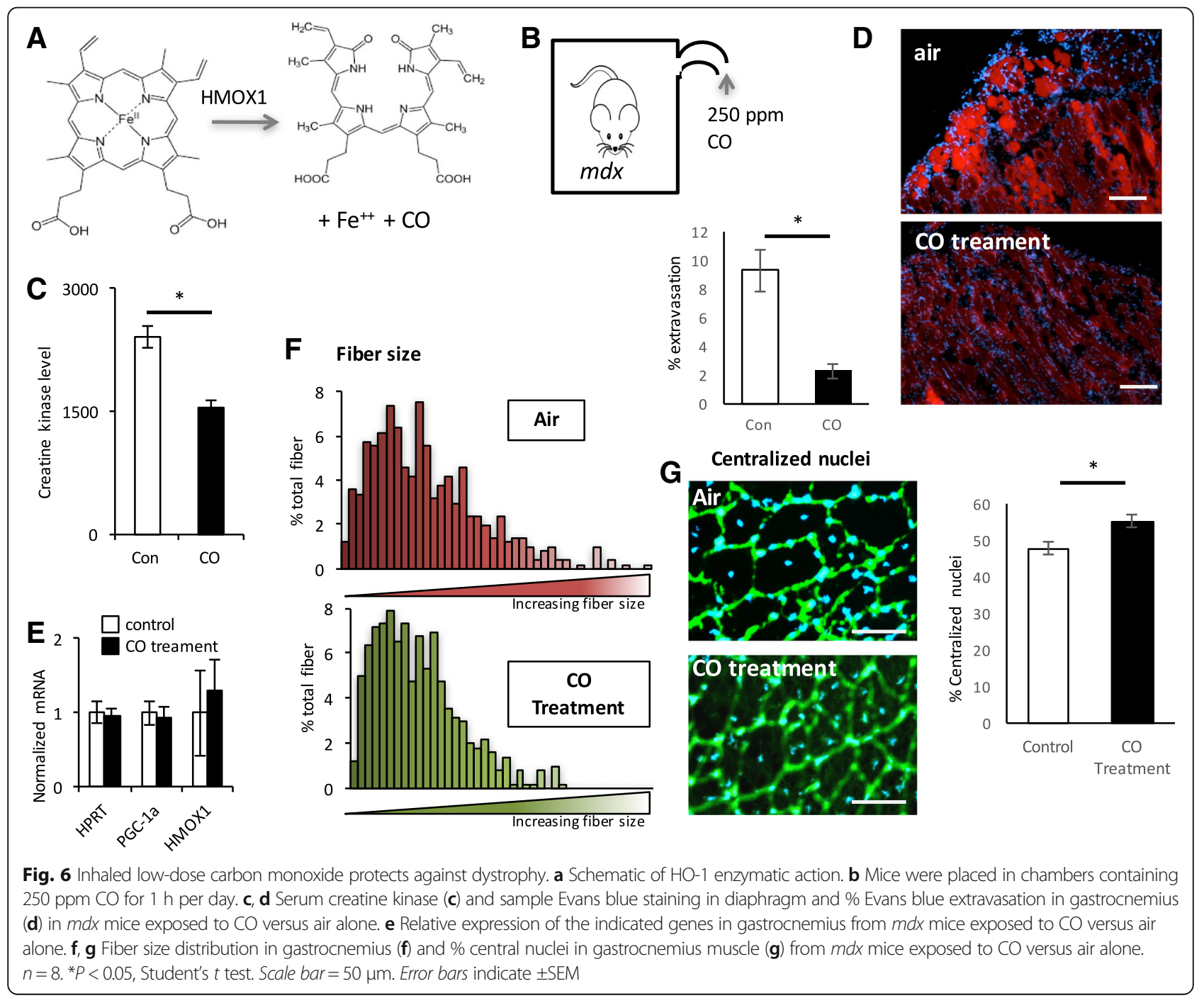




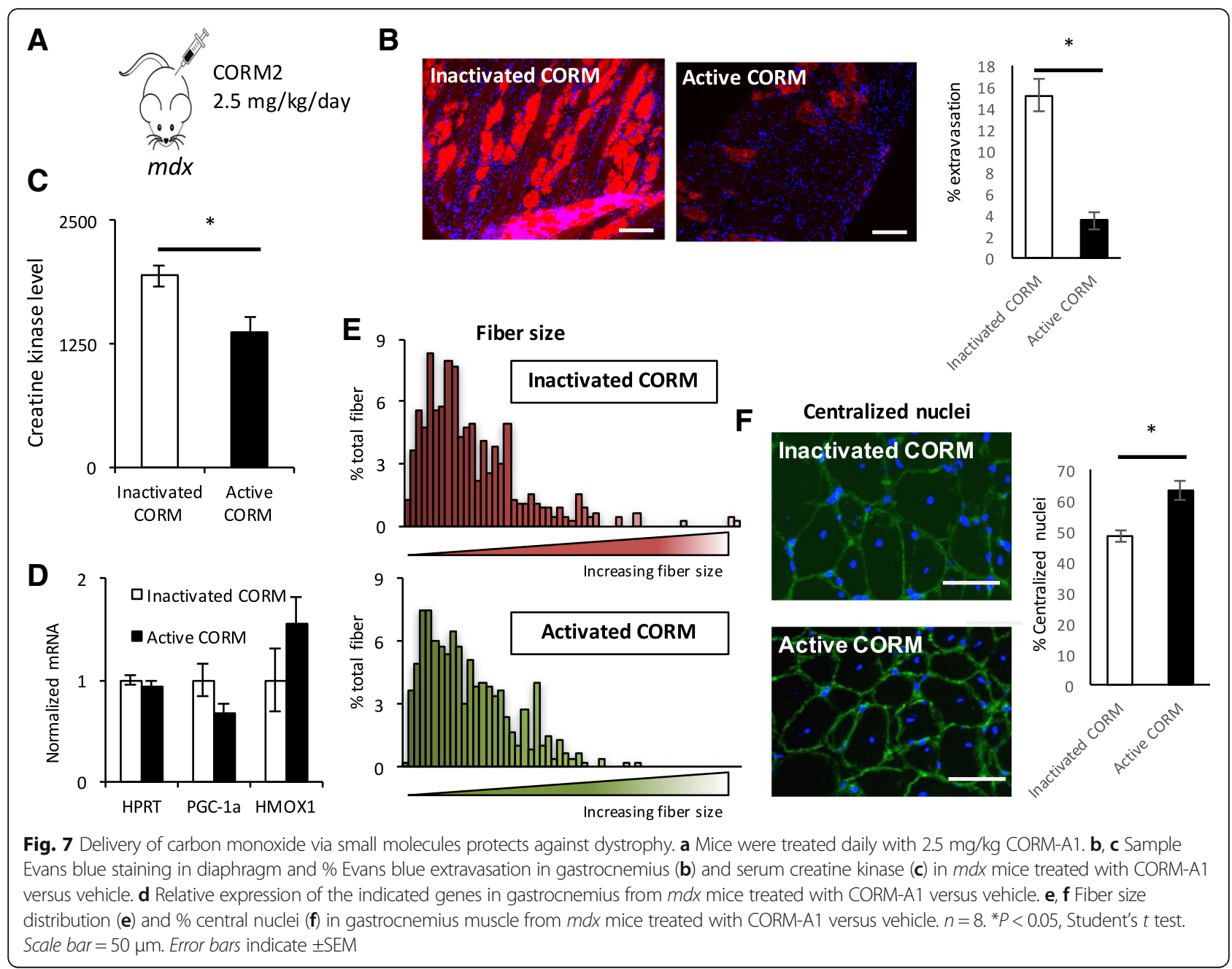

\section{Discussion}

We have leveraged here the transcriptional coactivator PGC- $1 \alpha$ to identify a novel pathway, involving the heme oxygenase enzyme and its enzymatic product carbon monoxide, as potent protective agents against dystrophy. One of the more appealing and transformative aspects of the work is its potential imminent translatability to the clinical setting. $\mathrm{CO}$ has an understandably nefarious public image, but the toxicity of $\mathrm{CO}$ occurs at doses orders of magnitude higher than those used here. Indeed, $\mathrm{CO}$ is produced endogenously by $\mathrm{HO}-1$ under normal physiology. The safety of $\mathrm{CO}$ in humans has been demonstrated in phase I dose escalation with no evidence of toxicity (clinicaltrials.gov NCT01050933, Otterbein personal communication, and [26]). Inhaled CO was also found to be safe in patients with COPD [34]. There are currently ongoing clinical trials of inhaled $\mathrm{CO}$ for treatment of adult and neonatal pulmonary hypertension (NCT01523548 and NCT01818843, respectively), ARDS (NCT02425579), post-operative ileus (NCT01050712), idiopathic pulmonary fibrosis (NCT01214187), and cardiac mitochondrial dysfunction (NCT01727167), extensively underscoring the accepted toxicity profile of CO. Importantly, in our experiments and in all of the above studies, inhaled $\mathrm{CO}$ is delivered only in intermittent doses, in our case $1 \mathrm{~h}$ a day. Translation to the clinic is therefore pragmatically feasible. Patients with DMD often have reduced respiratory capacity, in which case, the dose of delivery $\mathrm{CO}$ could be adjusted by measuring diffusing capacity of the lung for carbon monoxide (DLCO), a widely used clinical test. Alternatively, CO-releasing molecules (CO-RMs) may also serve as bioavailable alternatives to inhaled $\mathrm{CO}$, which would both facilitate delivery and allay fears of inhaling a gaseous therapeutic. Our work thus suggests that delivery of CO, either by inhalation of by CO-RMs, could be ready for clinical trials, and/or trials in large animals, to test for efficacy in the treatment of Duchenne muscular dystrophy, and perhaps other dystrophies as well.

How does heme oxygenase protect against dystrophy? Heme oxygenase degrades the cyclic prosthetic group heme, liberating ferrous iron and yielding biliverdin and 
gaseous $\mathrm{CO}$ (Fig. 3a). The protective effects of $\mathrm{HO}-1$ could therefore in principle stem from reductions of toxic heme or from beneficial effects elicited by biliverdin, iron, or CO. Mechanistically, we show here that CO alone is sufficient to protect against muscle dystrophy. Additional involvement by the other enzymatic products is also possible, as is suggested for example by the observation that treatment with CO or CORM both led to protection from damage, as determined by $\mathrm{CK}$ and EBD leak, but did not lead to larger fibers, in contrast to treatment with $\mathrm{HO}-1$ activators.

How CO itself protects against dystrophy is also of interest and will be the subject of future studies. One possibility is the observation that $\mathrm{CO}$ can, in some contexts, promote the formation of NO [26]. NO has been espoused as beneficial in models of dystrophy, perhaps in part via vasodilation and improved local ischemia $[3,4,35]$. Exposure to $\mathrm{CO}$ can also change cellular bioenergetics and increase oxygen consumption [36, 37] and can increase mitochondrial biogenesis in the muscle from humans and animals [38, 39]. $\mathrm{CO}$-induced mitochondrial biogenesis may thus contribute to protection against dystrophy and if so may constitute a positive feedback loop with PGC-1 $\alpha$ expression.

Although we have focused our attention here on heme oxygenase as a novel therapeutic opportunity, the antidystrophy protection that is afforded by PGC- $1 \alpha$ is likely multifactorial and is unlikely limited only to the induction of heme oxygenase. Because complete deletion of Hmox1 has diffuse effects, testing the relative contribution of the HO-1 system to PGC- $1 \alpha$-mediated protection will require introducing muscle-specific deletion of Hmox1 into transgenic PGC-1 $\alpha$ mice in the $m d x$ background.

\section{Conclusions}

In summary, our work highlights a new and actionable approach to the treatment of patients with DMD, including the appealing possibility that low-dose inhalation of $\mathrm{CO}$ could provide a novel and simple therapy for this devastating disease.

\footnotetext{
Abbreviations

CO: Carbon monoxide; CORM-A1: CO-releasing molecule A1;

DMD: Duchenne muscular dystrophy; HO: Heme oxygenase; MCK: Muscle creatine kinase; mdx: Dystrophin-deficient mice; NMJ: Neuromuscular junction; nNOS: Nitric oxide synthase; PGC-1a: Peroxisome proliferatoractivated receptor gamma coactivator $1-\alpha$; PGC-1 $\beta$ : Peroxisome proliferatoractivated receptor gamma coactivator $1-\beta$; TRE: tet-response element promoter; tTA: Tetracycline-dependent activator; VEGF: Vascular endothelial growth factor; WT: Wild-type/control
}

Acknowledgements

We thank Dr. Louis Kunkel for contributing samples from DMD patients.

\section{Funding}

The NHLBI (ZA and GCR), the Ellison Foundation (ZA), the MDA (ZA), and the NRSA (SR) supported this work.
Availability of data and materials

All data generated or analyzed during this study are included in this published article.

\section{Authors' contributions}

MCC designed and conducted the experiments and wrote and edited the manuscript. GCR, OZ, and LL contributed to conducting experiments. ZA, SD, and LEO contributed to the experimental design. ZA wrote and edited the manuscript. All authors read and approved the final manuscript.

\section{Competing interests}

The authors declare that they have no competing interests.

\section{Consent for publication}

Not applicable

\section{Ethics approval}

All animal experiments were performed according to the procedures approved by the Beth Israel Deaconess Medical Center's Institutional Animal Care and Use Committee.

\section{Author details}

${ }^{1}$ Cardiovascular Institute, Beth Israel Deaconess Medical Center, Boston, MA USA. ${ }^{2}$ Current address: Cardiovascular Institute, Massachusetts General Hospital, Boston, MA, USA. ${ }^{3}$ Present Address: Department of Medicine, University of Alabama at Birmingham, Birmingham, AL, USA. ${ }^{4}$ Department of Surgery, Beth Israel Deaconess Medical Center, Boston, MA, USA.

${ }^{5}$ Cardiovascular Institute and Institute Diabetes Obesity and Metabolism, Smilow Center for Translational Research, Perelman School of Medicine, University of Pennsylvania, 11th floor, 3400 Civic Blvd, Philadelphia 19104, PA, USA.

Received: 29 July 2016 Accepted: 18 November 2016

Published online: 28 November 2016

\section{References}

1. Guiraud S, et al. The pathogenesis and therapy of muscular dystrophies. Annu Rev Genomics Hum Genet. 2015;16:281-308.

2. Fairclough RJ, Bareja A, Davies KE. Progress in therapy for Duchenne muscular dystrophy. Exp Physiol. 2011;96(11):1101-13.

3. Brenman JE, et al. Nitric oxide synthase complexed with dystrophin and absent from skeletal muscle sarcolemma in Duchenne muscular dystrophy. Cell. 1995;82(5):743-52.

4. Kobayashi YM, et al. Sarcolemma-localized nNOS is required to maintain activity after mild exercise. Nature. 2008;456(7221):511-5.

5. Tabebordbar $\mathrm{M}$, et al. In vivo gene editing in dystrophic mouse muscle and muscle stem cells. Science. 2016;351(6271):407-11.

6. Nelson $\mathrm{CE}_{\text {, et }}$ al. In vivo genome editing improves muscle function in a mouse model of Duchenne muscular dystrophy. Science. 2016;351(6271):403-7.

7. Long C, et al. Postnatal genome editing partially restores dystrophin expression in a mouse model of muscular dystrophy. Science. 2016;351(6271):400-3.

8. Chan, MC, Arany Z. The many roles of PGC-1alpha in muscle-recent developments. Metabolism. 2014;63(4):441-51.

9. Handschin C, Spiegelman BM. Peroxisome proliferator-activated receptor gamma coactivator 1 coactivators, energy homeostasis, and metabolism. Endocr Rev. 2006;27(7):728-35.

10. Rowe GC, Jiang A, Arany Z. PGC-1 coactivators in cardiac development and disease. Circ Res. 2011;107(7):825-38.

11. Lin J, et al. Transcriptional co-activator PGC-1 alpha drives the formation of slow-twitch muscle fibres. Nature. 2002;418(6899):797-801.

12. Calvo JA, et al. Muscle-specific expression of PPAR\{gamma\} coactivator1 \{alpha\} improves exercise performance and increases peak oxygen uptake. J Appl Physiol. 2008;104(5):1304-12.

13. Handschin $\mathrm{C}$, et al. PGC-1alpha regulates the neuromuscular junction program and ameliorates Duchenne muscular dystrophy. Genes Dev. 2007:21(7):770-83

14. Chan MC, et al. Post-natal induction of PGC-1alpha protects against severe muscle dystrophy independently of utrophin. Skelet Muscle. 2014;4(1):2

15. Arany $Z$, et al. The transcriptional coactivator PGC-1 beta drives the formation of oxidative type IIX fibers in skeletal muscle. Cell Metab. 2007:5(1):35-46 
16. Russell LK, et al. Cardiac-specific induction of the transcriptional coactivator peroxisome proliferator-activated receptor gamma coactivator-1alpha promotes mitochondrial biogenesis and reversible cardiomyopathy in a developmental stage-dependent manner. Circ Res. 2004;94(4):525-33.

17. Rowe GC, et al. PGC-1alpha induces SPP1 to activate macrophages and orchestrate functional angiogenesis in skeletal muscle. Circ Res. 2014;115(5):504-17.

18. May $D$, et al. Transgenic system for conditional induction and rescue of chronic myocardial hibernation provides insights into genomic programs of hibernation. Proc Natl Acad Sci U S A. 2008;105(1):282-7.

19. Rowe GC, et al. Disconnecting mitochondrial content from respiratory chain capacity in PGC-1-deficient skeletal muscle. Endocr Rev. 2006;27(7):728-35.

20. St-Pierre J, et al. Bioenergetic analysis of peroxisome proliferator-activated receptor gamma coactivators 1alpha and 1beta (PGC-1alpha and PGC1beta) in muscle cells. J Biol Chem. 2003:278(29):26597-603.

21. Rowe GC, et al. PGC-1\{beta\} regulates angiogenesis in skeletal muscle. Am J Physiol Endocrinol Metab. 2011;301(1):E155-63.

22. Chinsomboon J, et al. The transcriptional coactivator PGC-1\{alpha\} mediates exercise-induced angiogenesis in skeletal muscle. Proc Natl Acad Sci U S A. 2009;106(50):21401-6.

23. Wagner BK, Arany Z. High-throughput real-time PCR for detection of geneexpression levels. Methods Mol Biol. 2009;486:167-75.

24. Arany $Z$, et al. HIF-independent regulation of VEGF and angiogenesis by the transcriptional coactivator PGC-1a. Nature. 2008;451:1008-12.

25. Rowe GC, et al. PGC-1alpha induces SPP1 to activate macrophages and orchestrate functional angiogenesis in skeletal muscle. Circ Res. 2014. in press.

26. Motterlini R, Otterbein LE. The therapeutic potential of carbon monoxide. Nat Rev Drug Discov. 2010;9(9):728-43.

27. Otterbein LE, Foresti R, Motterlini R. Heme oxygenase-1 and carbon monoxide in the heart: the balancing act between danger signaling and pro-survival. Circ Res. 2016;118(12):1940-59.

28. Ryter SW, Alam J, Choi AM. Heme oxygenase-1/carbon monoxide: from basic science to therapeutic applications. Physiol Rev. 2006;86(2):583-650.

29. Kawahara $G$, et al. Dystrophic muscle improvement in zebrafish via increased heme oxygenase signaling. Hum Mol Genet. 2014;23(7):1869-78.

30. Kawahara $G$, et al. Dystrophic muscle improvement in zebrafish via increased heme oxygenase signaling. Hum Mol Genet. 2015;24(15):4480-1.

31. Shan Y, et al. Role of Bach1 and Nrf2 in up-regulation of the heme oxygenase-1 gene by cobalt protoporphyrin. FASEB J. 2006;20(14):2651-3.

32. Yoshida T, et al. H(mox-1) constitutes an adaptive response to effect antioxidant cardioprotection: a study with transgenic mice heterozygous for targeted disruption of the heme oxygenase-1 gene. Circulation. 2001;103(12):1695-701.

33. Motterlini $\mathrm{R}$, et al. CORM-A1: a new pharmacologically active carbon monoxide-releasing molecule. FASEB J. 2005;19(2):284-6.

34. Bathoorn $\mathrm{E}$, et al. Anti-inflammatory effects of inhaled carbon monoxide in patients with COPD: a pilot study. Eur Respir J. 2007:30(6):1131-7.

35. Wehling M, Spencer MJ, Tidball JG. A nitric oxide synthase transgene ameliorates muscular dystrophy in mdx mice. J Cell Biol. 2001;155(1):123-31.

36. Schallner N, Otterbein LE. Friend or foe? Carbon monoxide and the mitochondria. Front Physiol. 2015;6:17

37. Wegiel B, et al. Carbon monoxide expedites metabolic exhaustion to inhibit tumor growth. Cancer Res. 2013;73(23):7009-21.

38. Rhodes MA, et al. Carbon monoxide, skeletal muscle oxidative stress, and mitochondrial biogenesis in humans. Am J Physiol Heart Circ Physiol. 2009;297(1):H392-9.

39. Pecorella SR, et al. The HO-1/CO system regulates mitochondrial-capillary density relationships in human skeletal muscle. Am J Physiol Lung Cell Mol Physiol. 2015;309(8):L857-71.

\section{Submit your next manuscript to BioMed Central and we will help you at every step:}

- We accept pre-submission inquiries

- Our selector tool helps you to find the most relevant journal

- We provide round the clock customer support

- Convenient online submission

- Thorough peer review

- Inclusion in PubMed and all major indexing services

- Maximum visibility for your research

Submit your manuscript at www.biomedcentral.com/submit
Biomed Central 